\title{
Dual Parametrik Eğrilerin Denklik Problemi
}

\section{Nurcan DEMIRCAN BEKAR ${ }^{1 *}$,Ömer PEKŞEN²}

${ }^{1}$ Türk Hava Kurumu Üniversitesi, Ankara, Türkiye

${ }^{2}$ Karadeniz Teknik Üniversitesi, Trabzon, Türkiye

\section{Öz}

$\mathbb{R}$ reel sayılar cismi ve $D=\left\{\left(a, a^{*}\right)=a+\varepsilon a^{*}, a, a^{*} \in \mathbb{R}, \varepsilon^{2}=0\right\} \quad$ dual cebir olsun. $D$ nin $D_{1}=\left\{\left(a, a^{*}\right), a \neq 0, a, a^{*} \in \mathbb{R}\right\}$ alt kümesi çarpma işlemine göre değişmeli bir grup oluşturur. Bir $A=a+\varepsilon a^{*} \in D_{1}$ elemanı ve $S: \mathbb{R}^{2} \rightarrow \mathbb{R}^{2}$ dönüşümü için $S(A)=S_{A}=\left(\begin{array}{cc}a & 0 \\ a^{*} & a\end{array}\right)$ olmak üzere; $I D_{1}^{+}=\left\{S_{A}=\left(\begin{array}{cc}a & 0 \\ a^{*} & a\end{array}\right), a \neq 0, a, a^{*} \in \mathbb{R}\right\} \quad$ ve $\quad I D_{1}^{-}=\left\{\left(\begin{array}{cc}a & 0 \\ a^{*} & a\end{array}\right)\left(\begin{array}{cc}1 & 0 \\ 0 & -1\end{array}\right), a \neq 0, a, a^{*} \in \mathbb{R}\right\} \quad$ kümelerini tanımlayalım. $I D_{1}=I D_{1}^{+} \cup I D_{1}^{-}$olsun. Ayrıca;

$\mathcal{M} I D_{1}^{+}=\left\{F: \mathbb{R}^{2} \rightarrow \mathbb{R}^{2}, F(B)=S_{A} B+C, A \in D_{1}, B, C \in \mathbb{R}^{2}\right\}$ ve $\mathcal{M} I I_{1}^{-}=\left\{F: \mathbb{R}^{2} \rightarrow \mathbb{R}^{2}, F(B)=\left(S_{A} W\right) B+C, A \in D_{1}, B, C \in \mathbb{R}^{2}, W=\left(\begin{array}{cc}1 & 0 \\ 0 & -1\end{array}\right)\right\}$ olmak üzere; $\mathcal{M} I D_{1}=\mathcal{M} I D_{1}^{+} \cup \mathcal{M} I D_{1}^{-}$şeklinde tanımlayalım. $T=(a, b) \quad \mathbb{R}$ 'de bir açık aralık olsun. Bir $\alpha: T \rightarrow \mathbb{R}^{2}, \forall t \in T$ için $\alpha(t)=(x(t), y(t))$ şeklindeki $C^{(2)}$-fonksiyonuna düzlemde bir parametrik eğri (yol) denir. $G$ bir grup olsun. $\forall t \in T$ ve bir $F \in G$ için $\beta(t)=F \alpha(t)$ eşitliği sağlanıyorsa $\alpha(t)$ ve $\beta(t)$ iki parametrik eğriye (yollara) $G$-denk eğriler denir. $\alpha(t) \sim \beta(t)$ ile gösterilir. Bu çalışma $\mathbb{R}^{2}$ Öklid uzayındaki parametrik eğriler (yollar) için $G=\mathcal{M} I D_{1}^{+}, \mathcal{M} I D_{1}$ gruplarına göre $G$-denklik probleminin çözümünü bulmaya yönelik bir çalışmadır.

Anahtar Kelimeler: Dual sayılar, parametrik eğri (yol), invariant.

\section{The Equivalence Problem Of Dual Parametric Curves}

\section{Abstract}

Let $\mathbb{R}$ be the field of real numbers and $D=\left\{\left(a, a^{*}\right)=a+\varepsilon a^{*}, a, a^{*} \in \mathbb{R}, \varepsilon^{2}=0\right\}$ be the algebra of dual numbers. The subset $D_{1}=\left\{\left(a, a^{*}\right), a \neq 0, a, a^{*} \in \mathbb{R}\right\}$ of $D$ is an abelian group with respect to the multiplication operation in the algebra $D$. For an element $A=a+\varepsilon a^{*} \in D_{1}$ and a transformation $S: \mathbb{R}^{2} \rightarrow \mathbb{R}^{2}$ where 
$S(A)=S_{A}=\left(\begin{array}{cc}a & 0 \\ a^{*} & a\end{array}\right), \quad$ we define the sets $I D_{1}^{+}=\left\{S_{A}=\left(\begin{array}{cc}a & 0 \\ a^{*} & a\end{array}\right), a \neq 0, a, a^{*} \in \mathbb{R}\right\} \quad$ and $I D_{1}^{-}=\left\{\left(\begin{array}{cc}a & 0 \\ a^{*} & a\end{array}\right)\left(\begin{array}{cc}1 & 0 \\ 0 & -1\end{array}\right), a \neq 0, a, a^{*} \in \mathbb{R}\right\}$. Let us denote $I D_{1}=I D_{1}^{+} \cup I D_{1}^{-}$. Moreover, we denote the set $\mathcal{M} I D_{1}=\mathcal{M} I D_{1}^{+} \cup \mathcal{M} I D_{1}^{-}$where

$\mathcal{M} I D_{1}^{+}=\left\{F: \mathbb{R}^{2} \rightarrow \mathbb{R}^{2}, F(B)=S_{A} B+C, A \in D_{1}, B, C \in \mathbb{R}^{2}\right\}$ and $\mathcal{M} I D_{1}^{-}=\left\{F: \mathbb{R}^{2} \rightarrow \mathbb{R}^{2}, F(B)=\left(S_{A} W\right) B+C, A \in D_{1}, B, C \in \mathbb{R}^{2}, W=\left(\begin{array}{cc}1 & 0 \\ 0 & -1\end{array}\right)\right\}$. Let $T=(a, b)$ be an open interval of $\mathbb{R}$. A $C^{(2)}$-function $\alpha: T \rightarrow \mathbb{R}^{2}$ for $\forall t \in T$ where, $\alpha(t)=(x(t), y(t))$ is called a parametrized curve (path) on the plane. Let $G$ be a group. Two parametric curves (paths) $\alpha(t)$ and $\beta(t)$ are called $G$ - equivalent if the equality $\beta(t)=F \alpha(t)$ is satisfied for an element $F \in G$ and all $t \in T$. Then, it is denoted by $\alpha(t) \sim \beta(t)$

This work is devoted to the solutions of problems of G-equivalence of parametric curves in Euclidean space $\mathbb{R}^{2}$ for the groups $G=\mathcal{M} I D_{1}^{+}, \mathcal{M} I D_{1}$.

Keywords: Dual numbers, parametric curve (path), invariant.

\section{Giriş}

İnvaryant teori ile ilgili çalışmalar 19. Yüzyılın ikinci yarısına dayanır ve invaryant teorinin gelişimi farklı alanları etkilemiştir. F. Klein, çalışması ile grup kavramının geometrilerin önemli yapı taşları olduğunu göstermiştir (Klein,1872). Bu çalışma verilen grup etkisi altında invaryant olan tüm özelliklerinden oluşmaktadır. Bunun yanında dual sayı kavramı William Kingdon Clifford tarafından ortaya çıkarılmış ve böylece yeni bir alanda bilimsel çalışmalar yapılmaya başlanmıştır. Bu çalışmanın temellerinden biri 2018 yılında yayınlanan 2-boyutlu Öklid Uzayı' nda Tüm Lineer Benzerlik Gruplar İçin Parametrik Eğrileri (yol) ve Eğrilerin Global İnvaryantları' çalışmasıdır (Khadjiev, vd. 2018). 2012 yılında 'Dual sayıların 2boyutlu Dual Geometriye Uygulanması' başlıklı tez çalışmasında 2-boyutlu dual düzlem geometrisinin temel gruplarından biri olan ve grupları için denklik problemleri incelenmiş, önemli tanımlar yapılmış ve bulgular elde edilmiştir (Tomar, 2012). Bu çalışmalar 1şığında dual cebir olmak üzere; nin alt kümesi çarpma işlemine göre değişmeli bir grup oluşturur. Buradan; $\mathcal{M} I D_{1}$ , $\mathcal{M} I D_{1}^{+}$grupları tanımlanmış bu gruplara göre iki parametrik eğrinin G-denklik kontrolü açısından basit ama etkili bir yöntemini bulmak amaçlanmıştır.

\section{2. $D$ Cebirinin Özellikleri}

\section{Tanım 2.1.}

$D=\left\{\left(a, a^{*}\right)=a+\varepsilon a^{*}, a, a^{*} \in \mathbb{R}, \varepsilon^{2}=0\right\}$

kümesi üzerinde toplama işlemi $\left(a, a^{*}\right)+\left(b, b^{*}\right)=\left(a+b, a^{*}+b^{*}\right)$ ile ve bir çarpma işlemi $\left(a, a^{*}\right) \times\left(b, b^{*}\right)=\left(a b, a b^{*}+a^{*} b\right)$ ile tanımlanırsa bu kümeye dual sayılar kümesi 
adı verilir. Bu küme bu iki işleme göre bir halka oluşturur. $A=\left(a, a^{*}\right) \in D$ dual sayısı $A=a+\varepsilon a^{*}$ şeklinde tek türlü yazılabilir. Yani; $A=\left(a, a^{*}\right)=a+\varepsilon a^{*} \quad$ dir. Ayrıca; $A=a+\varepsilon a^{*} \in D$ şeklindeki elemanlar $A=\left(\begin{array}{c}a \\ a^{*}\end{array}\right)$ olarak da yazılırlar.

Tanım 2.2. $A=a+\varepsilon a^{*} \in D$ dual sayısı olsun. $|A|=a$ olarak tanımlanır. Burada $\mid ., \quad \mathrm{D}$ kümesinde lineer operatördür.

Önerme 2.1. $A=a+\varepsilon a^{*} \in D$ ve $B=b+\varepsilon b^{*} \in D$ olmak üzere; $|A B|=|A||B|$ dir.

Önerme 2.2. $A=a+\varepsilon a^{*} \in D$ ve $\bar{A}=a-\varepsilon a^{*} \in D \quad$ 'ye $A$ dual sayisinın eşleniği denir ve $A+\bar{A}=2 a \in \mathbb{R}$, $|A|^{2}=A \bar{A}=a^{2} \in \mathbb{R}$.

Önerme 2.3. $A=a+\varepsilon a^{*} \in D, A^{-1}$ vardır. $\Leftrightarrow|A| \neq 0$. Ayrıca; $|A| \neq 0, A^{-1}=\frac{\bar{A}}{|A|^{2}}$ ve $\left|A^{-1}\right|=\frac{1}{|A|}$ dir.

$W=\left(\begin{array}{cc}1 & 0 \\ 0 & -1\end{array}\right)$ olmak üzere; $\bar{A}=W A=a-\varepsilon a^{*}$

Önerme 2.4. $A=a+\varepsilon a^{*} \in D$ olmak üzere; $|W A|=|A|$ dır. $\quad \forall A, B \in D \quad$ olmak üzere; $|(W A)(W B)|=|A||B|=|A B|$ dir.

İspat: $\quad A=a+\varepsilon a^{*} \in D$ olmak $\quad$ üzere; $|W A|=\left|\left(\begin{array}{cc}1 & 0 \\ 0 & -1\end{array}\right)\left(\begin{array}{c}a \\ a^{*}\end{array}\right)\right|=a=|A|$ olup istenen elde edilir. Ayrıca; $\forall A, B \in D$ olmak üzere; $|(W A)(W B)|=\left|\left(\left(\begin{array}{cc}1 & 0 \\ 0 & -1\end{array}\right)\left(\begin{array}{c}a \\ a^{*}\end{array}\right)\right)\left(\left(\begin{array}{cc}1 & 0 \\ 0 & -1\end{array}\right)\left(\begin{array}{c}b \\ b^{*}\end{array}\right)\right)\right|$ $=\left|\left(\begin{array}{c}a \\ a^{*}\end{array}\right)\left(\begin{array}{c}b \\ b^{*}\end{array}\right)\right|=\left|\left(\begin{array}{c}a b \\ a^{*} b^{*}\end{array}\right)\right|=a b=|A||B|=|A B|$ elde edilir.

3. $\mathbb{R}^{2}$ de Bir Parametrik Eğrinin (Yolun) $D_{1} \quad$ Grubuna Göre İnvaryantları

$D$ nin $\quad D_{1}=\left\{\left(a, a^{*}\right), a \neq 0, a, a^{*} \in \mathbb{R}\right\} \quad$ alt kümesi $\quad\left(a, a^{*}\right) \times\left(b, b^{*}\right)=\left(a b, a b^{*}+a^{*} b\right) \quad$ ile tanımlanan çarpma işlemine göre bir değişmeli grup oluşturur.

Bir $\quad A=a+\varepsilon a^{*} \in D_{1} \quad$ elemanı için $S: \mathbb{R}^{2} \rightarrow \mathbb{R}^{2} \quad$ dönüşümü için $S(A)=S_{A}=\left(\begin{array}{cc}a & 0 \\ a^{*} & a\end{array}\right)$ olmak üzere;

$I D^{+}{ }_{1}=\left\{S_{A}=\left(\begin{array}{cc}a & 0 \\ a^{*} & a\end{array}\right), a \neq 0, a, a^{*} \in \mathbb{R}\right\} \quad$ ve $I D^{-}=\left\{\left(\begin{array}{cc}a & 0 \\ a^{*} & a\end{array}\right)\left(\begin{array}{cc}1 & 0 \\ 0 & -1\end{array}\right), a \neq 0, a, a^{*} \in \mathbb{R}\right\}$

kümelerini tanımlayalım. $I D_{1}^{+} \cup I D_{1}^{-}$

kümesini $I D_{1}$ ile gösterelim. $D_{1}$ grubu $I D_{1}^{+}=\left\{\left(\begin{array}{cc}a & 0 \\ a^{*} & a\end{array}\right), a \neq 0, a, a^{*} \in \mathbb{R}\right\} \quad$ grubuna izomorftur. Öyle ki;

$$
\varphi: D_{1} \rightarrow I D_{1}^{+}, a \neq 0, H=a+\varepsilon a^{*} \in D_{1}
$$

$\varphi(H)=\varphi\left(a, a^{*}\right)=\left(\begin{array}{cc}a & 0 \\ a^{*} & a\end{array}\right)$ olarak tanımlanan $\varphi$ fonksiyonu bir grup izomorfizmasıdır. Aynı zamanda; $\forall B \in D$ olmak üzere; $S_{A} B=\left(\begin{array}{cc}a & 0 \\ a^{*} & a\end{array}\right)\left(\begin{array}{c}b \\ b^{*}\end{array}\right)=\left(\begin{array}{c}a b \\ a^{*} b+a b^{*}\end{array}\right)$ dir.

Tanım 3.1. $A, B \in D$ olmak üzere; $B=H A$ olacak şekilde bir $H \in D_{1}$ varsa $A$ ve $B$ elemanlarına $D_{1}$ - denktir denir ve $A \sim B$ ile gösterilir.

Tanım 3.2. $A=\left\{A_{1}, B_{1}\right\}$ ve $B=\left\{B_{1}, B_{2}\right\}$ öyle $\mathrm{ki} ; \quad B_{1}=H A_{1}, \quad B_{2}=H A_{2}$ olacak şekilde $H \in D_{1}$ varsa $A, B$ sistemlerine $D_{1}-$ denktir 
denir ve $\left\{A_{1}, A_{2}\right\} \stackrel{D_{1}}{\sim}\left\{B_{1}, B_{2}\right\}$ ile gösterilir.

Tanım 3.3. Bir $f: D^{2}=D \times D \rightarrow D$ fonksiyonuna $\forall H \in D_{1}$ ve $\forall A_{1}, A_{2} \in D$ için $f\left(H A_{1}, H A_{2}\right)=f\left(A_{1}, A_{2}\right)$ ise $D_{1}$ - invaryant fonksiyon denir.

Tanım 3.4. Bir $f: \mathbb{R}^{2} \times \mathbb{R}^{2} \rightarrow \mathbb{R}$ fonksiyonuna $\forall H \in I D_{1}^{+}, \forall A_{1}, A_{2} \in \mathbb{R}^{2}$ için $f\left(H A_{1}, H A_{2}\right)=f\left(A_{1}, A_{2}\right)$ ise $f \quad$ 'ye $I D_{1}^{+}-$ invaryant fonksiyon denir.

Önerme 3.1. $\varphi: D^{2}=D \times D \rightarrow D$ ve $f_{1}, f_{2}: \mathbb{R}^{2} \times \mathbb{R}^{2} \rightarrow \mathbb{R} \quad$ olmak üzere $\varphi\left(A_{1}, A_{2}\right)=f_{1}\left(A_{1}, A_{2}\right)+\varepsilon f_{2}\left(A_{1}, A_{2}\right)$

fonksiyonu $D_{1}$-invaryanttır. $\Leftrightarrow f_{1}, f_{2}$ $I D_{1}^{+}$-invaryanttır.

Tanım 3.5. $A=a+\varepsilon a^{*} \in D \quad B=b+\varepsilon b^{*} \in D$ olmak üzere; $\operatorname{det}\left(\begin{array}{cc}a & b \\ a^{*} & b^{*}\end{array}\right)=a b^{*}-a^{*} b=[A B]$ olarak gösterilir.

Önerme 3.2. $A, B \in D, \quad A=a+\varepsilon a^{*}$ ve $|A| \neq 0, B=b+\varepsilon b^{*}$ olsun. Bu takdirde

i) $B A^{-1}=\frac{|B|}{|A|}+\varepsilon \frac{[A B]}{|A|^{2}}$ ve $S_{B A^{-1}}=\left(\begin{array}{cc}\frac{|B|}{|A|} & 0 \\ \frac{[A B]}{|A|^{2}} & \frac{|B|}{|A|}\end{array}\right)$ dir.

ii) $\operatorname{det}\left(S_{B A^{-1}}\right)=\frac{|B|^{2}}{|A|^{2}} \operatorname{dir}$

ve $\operatorname{det}\left(S_{B A^{-1}}\right) \neq 0 \Leftrightarrow|B| \neq 0$ dir.

İspat: i) $|A| \neq 0$ olduğundan $a \neq 0$ dir.

Dolayısıyla; $\quad A^{-1} \quad$ vardır. $\quad A^{-1}=\frac{\bar{A}}{|A|^{2}}$. $\bar{A}=a-\varepsilon a^{*}$ kullanılarak
$B A^{-1}=\left(b+\varepsilon b^{*}\right) \frac{\left(a-\varepsilon a^{*}\right)}{a^{2}}=\frac{a b-\varepsilon b a^{*}+\varepsilon b^{*} a}{a^{2}}$ $=\frac{b}{a}+\frac{\varepsilon\left(a b^{*}-a^{*} b\right)}{a}=\frac{|B|}{|A|}+\varepsilon \frac{[A B]}{|A|^{2}}$ elde edilir.

ii) $|A| \neq 0$ olduğundan

$S_{B A^{-1}}=\left(\begin{array}{cc}\frac{|B|}{|A|} & 0 \\ \frac{[A B]}{|A|^{2}} & \frac{|B|}{|A|}\end{array}\right) \quad$ olup $\operatorname{det}\left(S_{B A^{-1}}\right)=\frac{|B|^{2}}{|A|^{2}}$ elde edilir. $\operatorname{det}\left(S_{B A^{-1}}\right) \neq 0 \Leftrightarrow|B|^{2} \neq 0$ dir. Buradan $|B| \neq 0$ dir.

Önerme 3.3. $A \in D_{1}, B \in D$ olmak üzere;

i) $\quad f: D^{2} \rightarrow D$

$$
(A, B) \rightarrow f(A, B)=\frac{B}{A}=B A^{-1}
$$

$D_{1}$-invaryanttur.

ii) $\quad g: D^{2} \rightarrow \mathbb{R}$

$$
(A, B) \rightarrow g(A, B)=\frac{|B|}{|A|}
$$

$I D_{1}^{+}$-invaryanttır.

iii) $\quad h: D^{2} \rightarrow \mathbb{R}$

$$
(A, B) \rightarrow h(A, B)=\frac{[A B]}{|A|^{2}}
$$

$I D_{1}^{+}$-invaryanttır.

iv) $\quad k: D^{2} \rightarrow \mathbb{R}$

$$
(A, B) \rightarrow k(A, B)=\frac{[A B]^{2}}{|A|^{4}}
$$

$I D_{1}^{+}$-invaryanttır.

İspat: i) $f: D^{2} \rightarrow D$ ve $f(A, B)=\frac{B}{A}$, $A \in D_{1}, B \in D$ olmak üzere; $H \in D_{1}$ alalım. $f$ fonksiyonunun $\quad D_{1}$-invaryantliğını 
gösterelim:

$A \in D_{1}, B \in D, H \in D_{1} \quad$ olmak üzere; $f(H A, H B)=\frac{H B}{H A}=\frac{B}{A}=f(A, B) \quad$ olup $\quad f$

fonksiyonu $D_{1}$-invaryanttır.

ii) ve iii) $\quad A \in D_{1}, B \in D$ olmak üzere;

$f(A, B)=\frac{B}{A}=B A^{-1}=\frac{|B|}{|A|}+\varepsilon \frac{[A B]}{|A|^{2}}$ ve

$f(H A, H B)=\frac{H B}{H A}=\frac{|H B|}{|H A|}+\varepsilon \frac{[(H A)(H B)]}{|H A|^{2}}$

olur.

Biliyoruz ki; $f$ fonksiyonu $D_{1}$-invaryanttır.

O halde; Önerme 2.1.' den $\frac{|B|}{|A|}$ ve $\frac{[A B]}{|A|^{2}}$ da

$I D_{1}^{+}$-invaryanttır.

iv) $\frac{[A B]}{|A|^{2}} I D_{1}^{+}$-invaryant ise; $\left(\frac{[A B]}{|A|^{2}}\right)^{2}$

$I D_{1}^{+}$-invaryanttır.

Tanım 3.6. $A \in D_{1}, B \in D$ olmak üzere; $f: D^{2} \rightarrow D, f\left(W A_{1}, W A_{2}\right)=f\left(A_{1}, A_{2}\right)$ ise $f$ 'ye $W$-invaryant denir.

Önerme 3.4. i) $\forall A=a+\varepsilon a^{*} \in D$ için $|W A|=|A|$ ve $|W A|^{2}=|A|^{2} \operatorname{dir}$.

ii) $A \in D_{1}, B \in D$ olmak üzere; $\frac{|W B|}{|W A|}=\frac{|B|}{|A|}$ dir.

iii) $A \in D_{1}, B \in D$ olmak üzere; $\frac{[(W A)(W B)]^{2}}{|W A|^{4}}=\frac{[A B]^{2}}{|A|^{4}} \operatorname{dir}$.

İspat: $i$ ) Önerme 2.4. ten açıktır.

ii) Önerme 2.4. ve $i$ ) 'den açıktır.

iii) $\forall A=a+\varepsilon a^{*} \in D$ için $\quad|W A|=|A| \quad$ ve $(\operatorname{det}(W))^{2}=1$ kullanılarak;

$$
\begin{aligned}
& \frac{[(W A)(W B)]^{2}}{|W A|^{4}}=\frac{(\operatorname{det}(W)[A B])^{2}}{|A|^{4}} \\
& =(\operatorname{det}(W))^{2} \frac{[A B]^{2}}{|A|^{4}}=\frac{[A B]^{2}}{|A|^{4}} \text { elde edilir. }
\end{aligned}
$$

Tanım 3.7. $A \in D_{1}, B \in D$ olmak üzere; $f(A, B)$ fonksiyonu $D_{1}$-invaryant ve $W$ invaryant ise $f(A, B)$ fonksiyonu $D_{1} \cup W$ invaryanttur.

Önerme 3.5. i) $\frac{|B|}{|A|}$ fonksiyonu

$D_{1} \cup W$-invaryanttır.

ii) $\frac{[A B]^{2}}{|A|^{4}}$ fonksiyonu $D_{1} \cup W$-invaryanttır.

İspat: $i$ ) Önerme 3.3. (ii) ve Önerme 3.4. (ii)' den açıktır.

ii) Önerme 3.3. (iv) ve Önerme 3.4. (iii)' den açıktır.

$G=I D_{1}^{+}$veya $I D_{1}$ olmak üzere;

Tanım 3.8. $A=\left(A_{1}, A_{2}\right) \in D_{1} \times D$, $B=\left(B_{1}, B_{2}\right) \in D_{1} \times D$ olmak üzere; $B_{1}=H A_{1}$, $B_{2}=H A_{2}$ olacak şekilde $H \in G$ varsa $A$ ile $B$ 'ye $G-$ denk denir ve $A \stackrel{\mathrm{G}}{\sim} B$ ile gösterilir. Tanım 3.9. Bir $f: D^{2}=D \times D \rightarrow D$ fonksiyonuna $\forall H \in D_{1}$ ve $\forall A_{1}, A_{2} \in D$ için $f\left(H A_{1}, H A_{2}\right)=f\left(A_{1}, A_{2}\right) \quad$ ise $G$-invaryant fonksiyon denir.

Tanım 3.10. $T=(a, b) \quad \mathbb{R}$ 'de bir açık aralık olsun. $\quad$ Bir $\quad \alpha: T \rightarrow \mathbb{R}^{2}, \forall t \in T$ için $\alpha(t)=(x(t), y(t)) \quad$ şeklindeki $\quad C^{(2)}$ fonksiyonuna düzlemde bir parametrik eğri ( $T$-yol) denir. $\alpha(t)$ bir $T$-yol ise $F \in G$ için $F \alpha(t)$ de $\mathbb{R}^{2}$ 'de bir $T$-yoldur.

Tanım 3.11. $G$ bir grup olsun. $\forall t \in T$ ve bir $F \in G$ için $\beta(t)=F \alpha(t)$ olsun. Bu takdirde; $\alpha(t)$ ve $\beta(t)$ parametrik eğrilerine 
$G$-denk ĕgriler denir ve $\quad \alpha(t) \stackrel{G}{\sim} \beta(t)$ ile gösterilir.

Tanım 3.12. $\forall F \in G, \quad \forall t \in T \quad$ için $\alpha(t), \beta(t), \ldots, \theta(t) \quad$ sonlu sayida $\quad T$-yollar

olmak üzere; eğer

$f(F \alpha(t), F \beta(t), \ldots, F \theta(t))$

$=f(\alpha(t), \beta(t), \ldots, \theta(t))$ sağlanıyorsa

$\alpha(t), \beta(t), \ldots, \theta(t) \quad T$-yollartn

fonksiyonuna $G$-invaryant denir.

$\alpha(t)=(x(t), y(t)) \quad \mathbb{R}^{2}$ 'de bir $T$-yol

olsun. $\alpha(t)$ 'nin 1. türevi $\alpha^{\prime}(t)=\left(x^{\prime}(t), y^{\prime}(t)\right)$

dir. $\quad \alpha(t)$ ve $\quad \alpha^{\prime}(t)$ 'nin determinantı $\operatorname{det}\left(\begin{array}{ll}x(t) & x^{\prime}(t) \\ y(t) & y^{\prime}(t)\end{array}\right)=\left[\alpha(t) \alpha^{\prime}(t)\right] \operatorname{dir}$.

$|\alpha(t)|=x(t)$.

Tanım 3.13. $\mathbb{R}^{2}$ 'de bir $T$-yol $\alpha(t)=(x(t), y(t))$ olsun. Eğer; $\forall t \in T$ için $|\alpha(t)| \neq 0$ ise yani $\forall t \in T$ için $\quad x(t) \neq 0$ ise $\alpha(t)$ 'ye $c$-regüler denir.

Teorem 3.1. $\alpha(t)=(x(t), y(t)) \mathbb{R}^{2}$ de bir $c$-regüler $T$-yol olsun.

i) $\frac{\alpha^{\prime}(t)}{\alpha(t)}$ fonksiyonu, $\mathbb{R}^{2}$ 'deki tüm c-regüler $\alpha(t) T$-yolların kümesi üzerinde

$D_{1}$-invaryanttır.

ii) $\frac{\left|\alpha^{\prime}(t)\right|}{|\alpha(t)|}$ fonksiyonu, $\mathbb{R}^{2}$ 'deki tüm c-regüler

$\alpha(t) T$-yolların kümesi üzerinde

$I D_{1}^{+}$-invaryanttır.

iii) $\frac{\left[\alpha(t) \alpha^{\prime}(t)\right]}{|\alpha(t)|^{2}}$ fonksiyonu, $\mathbb{R}^{2}$, deki tüm

c-regüler $\alpha(t) \quad T$-yolların kümesi üzerinde

$I D_{1}^{+}$-invaryanttır.

İspat:

$I D^{+}{ }_{1}=\left\{S_{A}=\left(\begin{array}{cc}a & 0 \\ a^{*} & a\end{array}\right), a \neq 0, a, a^{*} \in \mathbb{R}\right\} \mathrm{ve}$

Tanım 3.12 kullanılarak; i) $f(\alpha(t))=\frac{\alpha^{\prime}(t)}{\alpha(t)}$ fonksiyonunun

$D_{1}$-invaryant olduğunu gösterelim.

$F \in D_{1}$ olduğundan

$F \alpha(t)=S_{A} \alpha(t)=\left(\begin{array}{cc}a & 0 \\ a^{*} & a\end{array}\right)\left(\begin{array}{l}x(t) \\ y(t)\end{array}\right)$ yazılır.

$=\left(\begin{array}{c}a x(t) \\ a^{*} x(t)+a y(t)\end{array}\right)=a x(t)+\varepsilon\left(a^{*} x(t)+a y(t)\right)$

$(F \alpha(t))^{\prime}=a x^{\prime}(t)+\varepsilon\left(a^{*} x^{\prime}(t)+a y^{\prime}(t)\right) \operatorname{dir}$.

$f(F \alpha(t))=\frac{(F \alpha(t))^{\prime}}{(F \alpha(t))}=\frac{a x^{\prime}(t)+\varepsilon\left(a^{*} x^{\prime}(t)+a y^{\prime}(t)\right)}{a x(t)+\varepsilon\left(a^{*} x(t)+a y(t)\right)}$

$=\frac{\left(a x^{\prime}(t)+\varepsilon\left(a^{*} x^{\prime}(t)+a y^{\prime}(t)\right)\right)\left(a x(t)-\varepsilon\left(a^{*} x(t)+a y(t)\right)\right)}{\left(a x(t)+\varepsilon\left(a^{*} x(t)+a y(t)\right)\right)\left(a x(t)-\varepsilon\left(a^{*} x(t)+a y(t)\right)\right)}$

$=\frac{\left(a x^{\prime}(t)+\varepsilon a^{*} x^{\prime}(t)+\varepsilon a y^{\prime}(t)\right)\left(a x(t)-\varepsilon a^{*} x(t)-\varepsilon a y(t)\right)}{a^{2}(x(t))^{2}}$

$=\frac{a^{2} x^{\prime}(t) x(t)+\varepsilon a^{2}\left(y^{\prime}(t) x(t)-x^{\prime}(t) y(t)\right)}{a^{2}(x(t))^{2}}$

$=\frac{a^{2}\left(x^{\prime}(t) x(t)+\varepsilon\left(y^{\prime}(t) x(t)-x^{\prime}(t) y(t)\right)\right)}{a^{2}(x(t))^{2}}$

$=\frac{x^{\prime}(t) x(t)+\varepsilon\left(y^{\prime}(t) x(t)-x^{\prime}(t) y(t)\right)}{(x(t))^{2}}$

$\frac{x^{\prime}(t) x(t)+\varepsilon\left(y^{\prime}(t) x(t)-x^{\prime}(t) y(t)\right)}{(x(t))^{2}}$ ifadesinin

$\frac{(x(t)+\varepsilon y(t))^{\prime}}{(x(t)+\varepsilon y(t))}$ olduğu görülür. Şöyle ki;

$\frac{(x(t)+\varepsilon y(t))^{\prime}}{(x(t)+\varepsilon y(t))}=\frac{x^{\prime}(t)+\varepsilon y^{\prime}(t)}{x(t)+\varepsilon y(t)}$

$=\frac{\left(x^{\prime}(t)+\varepsilon y^{\prime}(t)\right)(x(t)-\varepsilon y(t))}{(x(t)+\varepsilon y(t))(x(t)-\varepsilon y(t))}$

elde edilir. 


$$
\begin{aligned}
f(F \alpha(t)) & =\frac{(F \alpha(t))^{\prime}}{(F \alpha(t))} \\
& =\frac{x^{\prime}(t) x(t)+\varepsilon\left(y^{\prime}(t) x(t)-x^{\prime}(t) y(t)\right)}{(x(t))^{2}} \\
& =\frac{\alpha^{\prime}(t)}{\alpha(t)}=f(\alpha(t)) \text { olup istenen elde }
\end{aligned}
$$

edilir ve $\frac{\alpha^{\prime}(t)}{\alpha(t)}$ fonksiyonu $D_{1}$-invaryanttır.

ii) $f(\alpha(t))=\frac{\left|\alpha^{\prime}(t)\right|}{|\alpha(t)|}$ fonksiyonunun

$I D_{1}^{+}$-invaryant olduğunu gösterelim.

$\alpha(t)=(x(t), y(t)) \quad$ için $\quad \alpha^{\prime}(t)=\left(x^{\prime}(t), y^{\prime}(t)\right)$

dir. $|\alpha(t)|=x(t)$ ve $\left|\alpha^{\prime}(t)\right|=x^{\prime}(t)$ dir.

$F \in I D_{1}^{+}$olduğundan

$$
\begin{aligned}
& F \alpha(t)=\left(\begin{array}{ll}
a & 0 \\
a^{*} & a
\end{array}\right)\left(\begin{array}{l}
x(t) \\
y(t)
\end{array}\right) \\
& =\left(\begin{array}{c}
a x(t) \\
a^{*} x(t)+a y(t)
\end{array}\right)=a x(t)+\varepsilon\left(a^{*} x(t)+a y(t)\right)
\end{aligned}
$$

ve $\quad|F \alpha(t)|=a x(t) \quad$ dir. Aynı zamanda

$F \alpha^{\prime}(t)=a x^{\prime}(t)+\varepsilon\left(a^{*} x^{\prime}(t)+a y^{\prime}(t)\right) \quad$ olup

$\left|F \alpha^{\prime}(t)\right|=a x^{\prime}(t)$ dir.

$f(F \alpha(t))=\frac{\left|F \alpha^{\prime}(t)\right|}{|F \alpha(t)|}=\frac{a x^{\prime}(t)}{a x(t)}$

$=\frac{x^{\prime}(t)}{x(t)}=\frac{\left|\alpha^{\prime}(t)\right|}{|\alpha(t)|}=f(\alpha(t))$ olup istenen elde

edilir. Yani $\frac{\left|\alpha^{\prime}(t)\right|}{|\alpha(t)|}$ fonksiyonu

$I D_{1}^{+}$-invaryanttır.

iii) $\quad f(\alpha(t))=\frac{\left[\alpha(t) \alpha^{\prime}(t)\right]}{|\alpha(t)|^{2}}$ fonksiyonunun

$I D_{1}^{+}$-invaryant olduğunu gösterelim.

$F \in I D_{1}^{+}$olduğundan $F \alpha(t)=\left(\begin{array}{cc}a & 0 \\ a^{*} & a\end{array}\right)\left(\begin{array}{l}x(t) \\ y(t)\end{array}\right)$ yazılır.

$$
=\left(\begin{array}{c}
a x(t) \\
a^{*} x(t)+a y(t)
\end{array}\right)=a x(t)+\varepsilon\left(a^{*} x(t)+a y(t)\right)
$$

ve $\quad|F \alpha(t)|=a x(t)$ dir. Aynı zamanda;

$F \alpha^{\prime}(t)=a x^{\prime}(t)+\varepsilon\left(a^{*} x^{\prime}(t)+a y^{\prime}(t)\right)$,

$\left|F \alpha^{\prime}(t)\right|=a x^{\prime}(t)$ ve

$f(\alpha(t))=\frac{\left[\alpha(t) \alpha^{\prime}(t)\right]}{|\alpha(t)|^{2}}=\frac{\operatorname{det}\left(\begin{array}{ll}x(t) & x^{\prime}(t) \\ y(t) & y^{\prime}(t)\end{array}\right)}{(x(t))^{2}} \operatorname{dir}$.
$f(F \alpha(t))=\frac{\left[(F \alpha(t))\left(F \alpha^{\prime}(t)\right)\right]}{|F \alpha(t)|^{2}}$

$=\frac{\operatorname{det}\left(\begin{array}{cc}a x(t) & a x^{\prime}(t) \\ a^{*} x(t)+a y(t) & a^{*} x^{\prime}(t)+a y^{\prime}(t)\end{array}\right)}{(a x(t))^{2}}$

$=\frac{a a^{*} x(t) x^{\prime}(t)+a^{2} x(t) y^{\prime}(t)-a a^{*} x^{\prime}(t) x(t)-a^{2} x^{\prime}(t) y(t)}{(a x(t))^{2}}$

$=\frac{a^{2}\left(x(t) y^{\prime}(t)-x^{\prime}(t) y(t)\right)}{a^{2}(x(t))^{2}}=\frac{x(t) y^{\prime}(t)-x^{\prime}(t) y(t)}{(x(t))^{2}}$

$=\frac{\left[\alpha(t) \alpha^{\prime}(t)\right]}{|\alpha(t)|^{2}}=f(\alpha(t))$

olup istenen elde edilir. Yani $\frac{\left[\alpha(t) \alpha^{\prime}(t)\right]}{|\alpha(t)|^{2}}$

fonksiyonu $I D_{1}^{+}$-invaryanttır.

Teorem 3.2. $\alpha(t)=(x(t), y(t)) \mathbb{R}^{2}$, de bir $c$ -

regüler $T$-yol olsun.

i) $\frac{\left|\alpha^{\prime}(t)\right|}{|\alpha(t)|}$ fonksiyonu, $\mathbb{R}^{2}$ 'deki tüm c-regüler

$\alpha(t) T$-yolların kümesi üzerinde

$I D_{1}$-invaryanttır.

ii) $\frac{\left[\alpha(t) \alpha^{\prime}(t)\right]^{2}}{|\alpha(t)|^{4}}$ fonksiyonu, $\mathbb{R}^{2}$ 'deki tüm

c-regüler $\alpha(t) \quad T$-yolların kümesi üzerinde

$I D_{1}$-invaryanttır. 
İspat: $\quad$ i) $f(\alpha(t))=\frac{\left|\alpha^{\prime}(t)\right|}{|\alpha(t)|} \quad$ fonksiyonunun $I D_{1}$-invaryant olduğunu gösterelim. $\alpha(t)=(x(t), y(t)) \quad$ olmak üzere; $\alpha^{\prime}(t)=\left(x^{\prime}(t), y^{\prime}(t)\right)$ ve $\left|\alpha^{\prime}(t)\right|=x^{\prime}(t)$ dir.

$F \in I D_{1}$ olduğundan;

$$
\begin{aligned}
& F \alpha(t)=\left(\begin{array}{cc}
a & 0 \\
a^{*} & a
\end{array}\right)\left(\begin{array}{cc}
1 & 0 \\
0 & -1
\end{array}\right)\left(\begin{array}{l}
x(t) \\
y(t)
\end{array}\right) \text { yazılır. } \\
& =\left(\begin{array}{cc}
a & 0 \\
a^{*} & -a
\end{array}\right)\left(\begin{array}{l}
x(t) \\
y(t)
\end{array}\right) \\
& =\left(\begin{array}{c}
a x(t) \\
a^{*} x(t)-a y(t)
\end{array}\right)=a x(t)+\varepsilon\left(a^{*} x(t)-a y(t)\right) \\
& \text { ve }|F \alpha(t)|=a x(t)
\end{aligned}
$$$$
F \alpha^{\prime}(t)=a x^{\prime}(t)+\varepsilon\left(a^{*} x^{\prime}(t)-a y^{\prime}(t)\right)
$$$$
\text { olup }
$$$$
\left|F \alpha^{\prime}(t)\right|=a x^{\prime}(t) \text { dir. }
$$

$$
\begin{aligned}
f(F \alpha(t)) & =\frac{\left|F \alpha^{\prime}(t)\right|}{|F \alpha(t)|}=\frac{a x^{\prime}(t)}{a x(t)}=\frac{x^{\prime}(t)}{x(t)} \\
& =\frac{\left|\alpha^{\prime}(t)\right|}{|\alpha(t)|}=f(\alpha(t))
\end{aligned}
$$

olup istenen elde edilir ve $\frac{\left|\alpha^{\prime}(t)\right|}{|\alpha(t)|}$ fonksiyonu $I D_{1}$-invaryanttır.

ii) $\quad f(\alpha(t))=\frac{\left[\alpha(t) \alpha^{\prime}(t)\right]^{2}}{|\alpha(t)|^{4}}$ fonksiyonunun $I D_{1}$-invaryant olduğunu gösterelim.

$F \in I D_{1}$ olduğundan;

$$
\begin{aligned}
& F \alpha(t)=\left(\begin{array}{cc}
a & 0 \\
a^{*} & a
\end{array}\right)\left(\begin{array}{cc}
1 & 0 \\
0 & -1
\end{array}\right)\left(\begin{array}{l}
x(t) \\
y(t)
\end{array}\right) \text { yazılır. } \\
& =\left(\begin{array}{cc}
a & 0 \\
a^{*} & -a
\end{array}\right)\left(\begin{array}{l}
x(t) \\
y(t)
\end{array}\right) \\
& =\left(\begin{array}{c}
a x(t) \\
a^{*} x(t)-a y(t)
\end{array}\right)=a x(t)+\varepsilon\left(a^{*} x(t)-a y(t)\right) . \\
& |F \alpha(t)|=a x(t) \\
& F \alpha^{\prime}(t)=a x^{\prime}(t)+\varepsilon\left(a^{*} x^{\prime}(t)-a y^{\prime}(t)\right) \mathrm{dir} .
\end{aligned}
$$

$$
f(\alpha(t))=\frac{\left[\alpha(t) \alpha^{\prime}(t)\right]^{2}}{|\alpha(t)|^{4}}=\frac{\left(\operatorname{det}\left(\begin{array}{ll}
x(t) & x^{\prime}(t) \\
y(t) & y^{\prime}(t)
\end{array}\right)\right)^{2}}{(x(t))^{4}}
$$$$
=\frac{\left(x(t) y^{\prime}(t)-x^{\prime}(t) y(t)\right)^{2}}{(x(t))^{4}} \text { dir. O halde; }
$$$$
f(F \alpha(t))=\frac{\left[(F \alpha(t))\left(F \alpha^{\prime}(t)\right)\right]^{2}}{|F \alpha(t)|^{4}}
$$$$
=\frac{\left(\operatorname{det}\left(\begin{array}{cc}
a x(t) & a x^{\prime}(t) \\
a^{*} x(t)-a y(t) & a^{*} x^{\prime}(t)-a y^{\prime}(t)
\end{array}\right)\right)^{2}}{(a x(t))^{4}}
$$$$
=\frac{\left(a a^{*} x(t) x^{\prime}(t)-a^{2} x(t) y^{\prime}(t)-a a^{*} x^{\prime}(t) x(t)+a^{2} x^{\prime}(t) y(t)\right)^{2}}{(a x(t))^{4}}
$$

$$
\begin{aligned}
& =\frac{a^{4}\left(x^{\prime}(t) y(t)-x(t) y^{\prime}(t)\right)^{2}}{a^{4}(x(t))^{4}} \\
& =\frac{\left(x^{\prime}(t) y(t)-x(t) y^{\prime}(t)\right)^{2}}{(x(t))^{4}}=f \alpha(t) \text { olup istenen }
\end{aligned}
$$

elde edilir ve $\frac{\left[\alpha(t) \alpha^{\prime}(t)\right]^{2}}{|\alpha(t)|^{4}}$ fonksiyonu

$I D_{1}$-invaryanttır.

\section{4. $\mathbb{R}^{2}$ 'de Bir Parametrik Ĕgrinin (Yolun) $\mathcal{M} I D_{1}^{+}, \mathcal{M} I D_{1}$ Gruplarına Göre İnvaryantları}

$$
\begin{aligned}
& \mathcal{M} I D_{1}^{+}=\left\{F: \mathbb{R}^{2} \rightarrow \mathbb{R}^{2}, F(B)=S_{A} B+C,\right. \\
& \left.\qquad A \in D_{1}, B, C \in \mathbb{R}^{2}\right\} \text { ve } \\
& \mathcal{M} I D_{1}^{-}=\left\{F: \mathbb{R}^{2} \rightarrow \mathbb{R}^{2}, F(B)=\left(S_{A} W\right) B+C,\right. \\
& \left.\qquad A \in D_{1}, B, C \in \mathbb{R}^{2}, W=\left(\begin{array}{cc}
1 & 0 \\
0 & -1
\end{array}\right)\right\} \\
& \text { olmak üzere; } \\
& \mathcal{M} I D_{1}=\mathcal{M} I D_{1}^{+} \cup \mathcal{M} I D_{1}^{-} \\
& \text {şeklinde tanımlayalım. } \\
& G=\mathcal{M} I D_{1}^{+}, \mathcal{M} I D_{1} \text { alalım. }
\end{aligned}
$$

Tanım 4.1. $\quad \mathbb{R}^{2}$ de bir $T$-yol 
$\alpha(t)=(x(t), y(t))$ olsun. Eğer; $\forall t \in T$ için $\left|\alpha^{\prime}(t)\right| \neq 0$ yani; $\forall t \in T$ için $x^{\prime}(t) \neq 0$ ise $\alpha(t)$ 'ye $d$-regüler denir.

$$
\alpha(t)=(x(t), y(t)) \quad \mathbb{R}^{2} \text { 'de bir } T \text {-yol }
$$

olsun. $\alpha(t)$ 'nin 1. türevi $\alpha^{\prime}(t)=\left(x^{\prime}(t), y^{\prime}(t)\right)$ ve $\alpha(t)$ 'nin 2. türevi $\alpha^{\prime \prime}(t)=\left(x^{\prime \prime}(t), y^{\prime \prime}(t)\right)$ olur. $\alpha^{\prime}(t)$ ve $\alpha^{\prime \prime}(t)$ için $\operatorname{det}\left(\begin{array}{ll}x^{\prime}(t) & x^{\prime \prime}(t) \\ y^{\prime}(t) & y^{\prime \prime}(t)\end{array}\right)=\left[\alpha^{\prime}(t) \alpha^{\prime \prime}(t)\right]$ ile gösterilir.

Teorem 4.1. $\alpha(t)=(x(t), y(t)) \mathbb{R}^{2}$ 'de bir $d$-regüler $T$-yol olsun.

i) $\frac{\alpha^{\prime \prime}(t)}{\alpha^{\prime}(t)}$ fonksiyonu, $\mathbb{R}^{2}$ 'deki tüm $d$-regüler $\alpha(t) T$-yolların kümesi üzerinde $\mathcal{M} I D_{1}^{+}$-invaryanttır.

ii) $\frac{\left|\alpha^{\prime \prime}(t)\right|}{\left|\alpha^{\prime}(t)\right|}$ fonksiyonu, $\mathbb{R}^{2}$, deki tüm $d$-regüler $\alpha(t) \quad T$-yolların kümesi üzerinde $\mathcal{M} I D_{1}^{+}$-invaryanttır.

iii) $\frac{\left[\alpha^{\prime}(t) \alpha^{\prime \prime}(t)\right]}{\left|\alpha^{\prime}(t)\right|^{2}}$ fonksiyonu, $\mathbb{R}^{2}$, deki tüm $d$-regüler $\alpha(t) \quad T$-yolların kümesi üzerinde $\mathcal{M} I D_{1}^{+}$-invaryanttur.

İspat: i) $f(\alpha(t))=\frac{\alpha^{\prime \prime}(t)}{\alpha^{\prime}(t)}$ fonksiyonunun $\mathcal{M} I D_{1}^{+}$-invaryant olduğunu gösterelim.

$F \in \mathcal{M} I D_{1}^{+}$olduğundan

$$
\begin{aligned}
& \begin{aligned}
F \alpha(t) & =\left(\begin{array}{ll}
a & 0 \\
a^{*} & a
\end{array}\right)\left(\begin{array}{l}
x(t) \\
y(t)
\end{array}\right)+\left(\begin{array}{l}
c_{1} \\
c_{2}
\end{array}\right) \\
& =\left(\begin{array}{c}
a x(t)+c_{1} \\
a^{*} x(t)+a y(t)+c_{2}
\end{array}\right) \\
& =a x(t)+c_{1}+\varepsilon\left(a^{*} x(t)+a y(t)+c_{2}\right)
\end{aligned} \\
& (F \alpha(t))^{\prime}=a x^{\prime}(t)+\varepsilon\left(a^{*} x^{\prime}(t)+a y^{\prime}(t)\right) \mathrm{ve} \\
& (F \alpha(t))^{\prime \prime}=a x^{\prime \prime}(t)+\varepsilon\left(a^{*} x^{\prime \prime}(t)+a y^{\prime \prime}(t)\right) \mathrm{dir} .
\end{aligned}
$$

$$
f(F \alpha(t))=\frac{(F \alpha(t))^{\prime \prime}}{(F \alpha(t))^{\prime}}=\frac{a x^{\prime \prime}(t)+\varepsilon\left(a^{*} x^{\prime \prime}(t)+a y^{\prime \prime}(t)\right)}{a x^{\prime}(t)+\varepsilon\left(a^{*} x^{\prime}(t)+a y^{\prime}(t)\right)}
$$

$$
=\frac{\left(a x^{\prime \prime}(t)+\varepsilon\left(a^{*} x^{\prime \prime}(t)+a y^{\prime \prime}(t)\right)\right)\left(a x^{\prime}(t)-\varepsilon\left(a^{*} x^{\prime}(t)+a y^{\prime}(t)\right)\right)}{\left(a x^{\prime}(t)+\varepsilon\left(a^{*} x^{\prime}(t)+a y^{\prime}(t)\right)\right)\left(a x^{\prime}(t)-\varepsilon\left(a^{*} x^{\prime}(t)+a y^{\prime}(t)\right)\right)}
$$$$
=\frac{\left(a x^{\prime \prime}(t)+\varepsilon a^{*} x^{\prime \prime}(t)+\varepsilon a y^{\prime \prime}(t)\right)\left(a x^{\prime}(t)-\varepsilon a^{*} x^{\prime}(t)-\varepsilon a y^{\prime}(t)\right)}{a^{2}\left(x^{\prime}(t)\right)^{2}}
$$

$=\frac{a^{2} x^{\prime \prime}(t) x^{\prime}(t)+\varepsilon a^{2}\left(y^{\prime \prime}(t) x^{\prime}(t)-x^{\prime \prime}(t) y^{\prime}(t)\right)}{a^{2}\left(x^{\prime}(t)\right)^{2}}$

$$
=\frac{a^{2}\left(x^{\prime \prime}(t) x^{\prime}(t)+\varepsilon\left(y^{\prime \prime}(t) x^{\prime}(t)-x^{\prime \prime}(t) y^{\prime}(t)\right)\right)}{a^{2}\left(x^{\prime}(t)\right)^{2}}
$$

$=\frac{x^{\prime \prime}(t) x^{\prime}(t)+\varepsilon\left(y^{\prime \prime}(t) x^{\prime}(t)-x^{\prime \prime}(t) y^{\prime}(t)\right)}{\left(x^{\prime}(t)\right)^{2}}$

$$
\frac{x^{\prime \prime}(t) x^{\prime}(t)+\varepsilon\left(y^{\prime \prime}(t) x^{\prime}(t)-x^{\prime \prime}(t) y^{\prime}(t)\right)}{\left(x^{\prime}(t)\right)^{2}}
$$

ifadenin $\frac{(x(t)+\varepsilon y(t))^{\prime \prime}}{(x(t)+\varepsilon y(t))^{\prime}} \quad$ olduğu görülür.

Şöyle ki;

$$
\begin{aligned}
& \frac{(x(t)+\varepsilon y(t))^{\prime \prime}}{(x(t)+\varepsilon y(t))^{\prime}}=\frac{x^{\prime \prime}(t)+\varepsilon y^{\prime \prime}(t)}{x^{\prime}(t)+\varepsilon y^{\prime}(t)} \\
& =\frac{\left(x^{\prime \prime}(t)+\varepsilon y^{\prime \prime}(t)\right)\left(x^{\prime}(t)-\varepsilon y^{\prime}(t)\right)}{\left(x^{\prime}(t)+\varepsilon y^{\prime}(t)\right)\left(x^{\prime}(t)-\varepsilon y^{\prime}(t)\right)}
\end{aligned}
$$

olup dolayısıyla;

$$
\begin{aligned}
& f(F \alpha(t))=\frac{(F \alpha(t))^{\prime \prime}}{(F \alpha(t))^{\prime}} \\
& =\frac{x^{\prime \prime}(t) x^{\prime}(t)+\varepsilon\left(y^{\prime \prime}(t) x^{\prime}(t)-x^{\prime \prime}(t) y^{\prime}(t)\right)}{\left(x^{\prime}(t)\right)^{2}}
\end{aligned}
$$

$=\frac{\alpha^{\prime \prime}(t)}{\alpha^{\prime}(t)}=f(\alpha(t))$ olup istenen elde edilir ve $\frac{\alpha^{\prime \prime}(t)}{\alpha^{\prime}(t)}$ fonksiyonu $\mathcal{M} I D_{1}^{+}$-invaryanttır.

ii) $f(\alpha(t))=\frac{\left|\alpha^{\prime \prime}(t)\right|}{\left|\alpha^{\prime}(t)\right|}$ fonksiyonunun 
$\mathcal{M} I D_{1}^{+}$-invaryant olduğunu gösterelim.

$\alpha(t)=(x(t), y(t)) \quad$ olmak üzere;

$\alpha^{\prime}(t)=\left(x^{\prime}(t), y^{\prime}(t)\right) \quad$ ve $\quad \alpha^{\prime \prime}(t)=\left(x^{\prime \prime}(t), y^{\prime \prime}(t)\right)$

dir.

$\left|\alpha^{\prime \prime}(t)\right|=x^{\prime \prime}(t)$ ve $\left|\alpha^{\prime}(t)\right|=x^{\prime}(t)$ dir.

$F \in \mathcal{M} I D_{1}^{+}$olduğundan

$f(\alpha(t))=\frac{\left[\alpha^{\prime}(t) \alpha^{\prime \prime}(t)\right]}{\left|\alpha^{\prime}(t)\right|^{2}}=\frac{\operatorname{det}\left(\begin{array}{ll}x^{\prime}(t) & x^{\prime \prime}(t) \\ y^{\prime}(t) & y^{\prime \prime}(t)\end{array}\right)}{\left(x^{\prime}(t)\right)^{2}}$

dir.

$$
\begin{aligned}
F \alpha(t) & =\left(\begin{array}{ll}
a & 0 \\
a^{*} & a
\end{array}\right)\left(\begin{array}{l}
x(t) \\
y(t)
\end{array}\right)+\left(\begin{array}{l}
c_{1} \\
c_{2}
\end{array}\right) \\
& =\left(\begin{array}{c}
a x(t)+c_{1} \\
a^{*} x(t)+a y(t)+c_{2}
\end{array}\right) \\
& =a x(t)+c_{1}+\varepsilon\left(a^{*} x(t)+a y(t)+c_{2}\right)
\end{aligned}
$$

$F \alpha^{\prime}(t)=a x^{\prime}(t)+\varepsilon\left(a^{*} x^{\prime}(t)+a y^{\prime}(t)\right) \quad$ olup

$f(F \alpha(t))=\frac{\left[\left(F \alpha^{\prime}(t)\right)\left(F \alpha^{\prime \prime}(t)\right)\right]}{\left|F \alpha^{\prime}(t)\right|^{2}}$

$=\frac{\operatorname{det}\left(\begin{array}{cc}a x^{\prime}(t) & a x^{\prime \prime}(t) \\ a^{*} x^{\prime}(t)+a y^{\prime}(t) & a^{*} x^{\prime \prime}(t)+a y^{\prime \prime}(t)\end{array}\right)}{\left(a x^{\prime}(t)\right)^{2}}$

$=\frac{a a^{*} x^{\prime}(t) x^{\prime \prime}(t)+a^{2} x^{\prime}(t) y^{\prime \prime}(t)-a a^{*} x^{\prime \prime}(t) x^{\prime}(t)-a^{2} x^{\prime \prime}(t) y^{\prime}(t)}{\left(a x^{\prime}(t)\right)^{2}}$

$\left|F \alpha^{\prime}(t)\right|=a x^{\prime}(t)$ dir.

$F \alpha^{\prime \prime}(t)=a x^{\prime \prime}(t)+\varepsilon\left(a^{*} x^{\prime \prime}(t)+a y^{\prime \prime}(t)\right) \quad$ olup

$\left|F \alpha^{\prime \prime}(t)\right|=a x^{\prime \prime}(t)$ dir.

olup $=\frac{a^{2}\left(x^{\prime}(t) y^{\prime \prime}(t)-x^{\prime \prime}(t) y^{\prime}(t)\right)}{a^{2}\left(x^{\prime}(t)\right)^{2}}=\frac{x^{\prime}(t) y^{\prime \prime}(t)-x^{\prime \prime}(t) y^{\prime}(t)}{\left(x^{\prime}(t)\right)^{2}}$

$f(F \alpha(t))=\frac{\left|F \alpha^{\prime \prime}(t)\right|}{\left|F \alpha^{\prime}(t)\right|}=\frac{a x^{\prime \prime}(t)}{a x^{\prime}(t)}=\frac{x^{\prime \prime}(t)}{x^{\prime}(t)}=\frac{\left|\alpha^{\prime \prime}(t)\right|}{\left|\alpha^{\prime}(t)\right|}=f(\alpha(t)) \quad=\frac{\left[\alpha^{\prime}(t) \alpha^{\prime \prime}(t)\right]}{\left|\alpha^{\prime}(t)\right|^{2}}=f \alpha(t)$

olup istenen elde edilir ve $\frac{\left|\alpha^{\prime \prime}(t)\right|}{\left|\alpha^{\prime}(t)\right|}$ fonksiyonu olup istenen elde edilir ve $\frac{\left[\alpha^{\prime}(t) \alpha^{\prime \prime}(t)\right]}{\left|\alpha^{\prime}(t)\right|^{2}}$

$\mathcal{M} I D_{1}^{+}$-invaryanttır.

iii) $f(\alpha(t))=\frac{\left[\alpha^{\prime}(t) \alpha^{\prime \prime}(t)\right]}{\left|\alpha^{\prime}(t)\right|^{2}}$ fonksiyonunun $\mathcal{M}$ fonksiyonu $\mathcal{M} I D_{1}^{+}$-invaryanttır.

$I D_{1}^{+}$-invaryant olduğunu gösterelim.

$F \in \mathcal{M} I D_{1}^{+}$olduğundan

$$
\begin{aligned}
F \alpha(t) & =\left(\begin{array}{ll}
a & 0 \\
a^{*} & a
\end{array}\right)\left(\begin{array}{l}
x(t) \\
y(t)
\end{array}\right)+\left(\begin{array}{l}
c_{1} \\
c_{2}
\end{array}\right) \\
& =\left(\begin{array}{c}
a x(t)+c_{1} \\
a^{*} x(t)+a y(t)+c_{2}
\end{array}\right) \\
& =a x(t)+c_{1}+\varepsilon\left(a^{*} x(t)+a y(t)+c_{2}\right) \\
F \alpha^{\prime}(t) & =a x^{\prime}(t)+\varepsilon\left(a^{*} x^{\prime}(t)+a y^{\prime}(t)\right), \\
F \alpha^{\prime \prime}(t) & =a x^{\prime \prime}(t)+\varepsilon\left(a^{*} x^{\prime \prime}(t)+a y^{\prime \prime}(t)\right), \\
\left|F \alpha^{\prime}(t)\right| & =a x^{\prime}(t) \text { ve }
\end{aligned}
$$

Teorem 4.2. $\alpha(t)=(x(t), y(t)) \mathbb{R}^{2}$ 'de bir $d$-regüler $T$-yol olsun.

i) $\frac{\left|\alpha^{\prime \prime}(t)\right|}{\left|\alpha^{\prime}(t)\right|}$ fonksiyonu, $\mathbb{R}^{2}$ 'deki tüm

$d$-regüler $\alpha(t) T$-yolların kümesi üzerinde $\mathcal{M} I D_{1}$-invaryanttur.

ii) $\frac{\left[\alpha^{\prime}(t) \alpha^{\prime \prime}(t)\right]^{2}}{\left|\alpha^{\prime}(t)\right|^{4}}$ fonksiyonu, $\mathbb{R}^{2}$, deki tüm $d$-regüler $\alpha(t) T$-yolların kümesi üzerinde $\mathcal{M} I D_{1}$-invaryanttır.

İspat: i) $f(\alpha(t))=\frac{\left|\alpha^{\prime \prime}(t)\right|}{\left|\alpha^{\prime}(t)\right|}$ fonksiyonunun $\mathcal{M} I D_{1}$-invaryant olduğunu gösterelim.

$\begin{array}{llll}\alpha(t)=(x(t), y(t)) & \text { olmak üzere; } \\ \alpha^{\prime}(t)=\left(x^{\prime}(t), y^{\prime}(t)\right) & \text { ve } & \alpha^{\prime \prime}(t)=\left(x^{\prime \prime}(t), y^{\prime \prime}(t)\right)\end{array}$ 
dir.

$\left|\alpha^{\prime \prime}(t)\right|=x^{\prime \prime}(t)$ ve $\left|\alpha^{\prime}(t)\right|=x^{\prime}(t)$ dir.

$F \in \mathcal{M} I D_{1}$ olduğundan;

$$
\begin{aligned}
F \alpha(t) & =\left(\begin{array}{cc}
a & 0 \\
a^{*} & a
\end{array}\right)\left(\begin{array}{cc}
1 & 0 \\
0 & -1
\end{array}\right)\left(\begin{array}{l}
x(t) \\
y(t)
\end{array}\right)+\left(\begin{array}{l}
c_{1} \\
c_{2}
\end{array}\right) \\
& =\left(\begin{array}{cc}
a & 0 \\
a^{*} & -a
\end{array}\right)\left(\begin{array}{l}
x(t) \\
y(t)
\end{array}\right)+\left(\begin{array}{l}
c_{1} \\
c_{2}
\end{array}\right) \\
& =\left(\begin{array}{c}
a x(t)+c_{1} \\
a^{*} x(t)-a y(t)+c_{2}
\end{array}\right) \\
& =a x(t)+c_{1}+\varepsilon\left(a^{*} x(t)-a y(t)+c_{2}\right) \\
F \alpha^{\prime}(t) & =a x^{\prime}(t)+\varepsilon\left(a^{*} x^{\prime}(t)-a y^{\prime}(t)\right)
\end{aligned}
$$

olup

$f(\alpha(t))=\frac{\left[\alpha^{\prime}(t) \alpha^{\prime \prime}(t)\right]^{2}}{\left|\alpha^{\prime}(t)\right|^{4}}=\frac{\left(\operatorname{det}\left(\begin{array}{ll}x^{\prime}(t) & x^{\prime \prime}(t) \\ y^{\prime}(t) & y^{\prime \prime}(t)\end{array}\right)\right)^{2}}{\left(x^{\prime}(t)\right)^{4}}$

$\left|F \alpha^{\prime}(t)\right|=a x^{\prime}(t)$ dir.

$F \alpha^{\prime \prime}(t)=a x^{\prime \prime}(t)+\varepsilon\left(a^{*} x^{\prime \prime}(t)-a y^{\prime \prime}(t)\right)$

olup

$=\frac{\left(x^{\prime}(t) y^{\prime \prime}(t)-x^{\prime \prime}(t) y^{\prime}(t)\right)^{2}}{\left(x^{\prime}(t)\right)^{4}}$ dir. O halde;

$f(F \alpha(t))=\frac{\left[\left(F \alpha^{\prime}(t)\right)\left(F \alpha^{\prime \prime}(t)\right)\right]^{2}}{\left|F \alpha^{\prime}(t)\right|^{4}}$

$$
=\frac{\left(\operatorname{det}\left(\begin{array}{cc}
a x^{\prime}(t) & a x^{\prime \prime}(t) \\
a^{*} x^{\prime}(t)-a y^{\prime}(t) & a^{*} x^{\prime \prime}(t)-a y^{\prime \prime}(t)
\end{array}\right)\right)^{2}}{\left(a x^{\prime}(t)\right)^{4}}
$$

$\left|F \alpha^{\prime \prime}(t)\right|=a x^{\prime \prime}(t)$ dir.

$f(F \alpha(t))=\frac{\left|F \alpha^{\prime \prime}(t)\right|}{\left|F \alpha^{\prime}(t)\right|}=\frac{a x^{\prime \prime}(t)}{a x^{\prime}(t)}=\frac{x^{\prime \prime}(t)}{x^{\prime}(t)}=\frac{\left|\alpha^{\prime \prime}(t)\right|}{\left|\alpha^{\prime}(t)\right|}$

$=\frac{a^{4}\left(x^{\prime \prime}(t) y^{\prime}(t)-x^{\prime}(t) y^{\prime \prime}(t)\right)^{2}}{a^{4}\left(x^{\prime}(t)\right)^{4}}$

$$
=f(\alpha(t))
$$

olup istenen elde edilir ve $\frac{\left|\alpha^{\prime \prime}(t)\right|}{\left|\alpha^{\prime}(t)\right|}$ fonksiyonu $\mathcal{M} I D_{1}$-invaryanttır.

ii) $\quad f(\alpha(t))=\frac{\left[\alpha^{\prime}(t) \alpha^{\prime \prime}(t)\right]^{2}}{\left|\alpha^{\prime}(t)\right|^{4}}$ fonksiyonunun $\mathcal{M} I D_{1}$-invaryant olduğunu gösterelim.

$F \in \mathcal{M} I D_{1}$ olduğundan;

$$
\begin{aligned}
F \alpha(t) & =\left(\begin{array}{cc}
a & 0 \\
a^{*} & a
\end{array}\right)\left(\begin{array}{cc}
1 & 0 \\
0 & -1
\end{array}\right)\left(\begin{array}{l}
x(t) \\
y(t)
\end{array}\right)+\left(\begin{array}{l}
c_{1} \\
c_{2}
\end{array}\right) \\
& =\left(\begin{array}{cc}
a & 0 \\
a^{*} & -a
\end{array}\right)\left(\begin{array}{l}
x(t) \\
y(t)
\end{array}\right)+\left(\begin{array}{l}
c_{1} \\
c_{2}
\end{array}\right) \\
& =\left(\begin{array}{c}
a x(t)+c_{1} \\
a^{*} x(t)-a y(t)+c_{2}
\end{array}\right) \\
& =a x(t)+c_{1}+\varepsilon\left(a^{*} x(t)-a y(t)+c_{2}\right)
\end{aligned}
$$

$F \alpha^{\prime}(t)=a x^{\prime}(t)+\varepsilon\left(a^{*} x^{\prime}(t)-a y^{\prime}(t)\right)$,

$F \alpha^{\prime \prime}(t)=a x^{\prime \prime}(t)+\varepsilon\left(a^{*} x^{\prime \prime}(t)-a y^{\prime \prime}(t)\right)$,

$$
=\frac{\left(x^{\prime \prime}(t) y^{\prime}(t)-x^{\prime}(t) y^{\prime \prime}(t)\right)^{2}}{\left(x^{\prime}(t)\right)^{4}}=f \alpha(t) \text { olup }
$$

istenen elde edilir ve $\frac{\left[\alpha^{\prime}(t) \alpha^{\prime \prime}(t)\right]^{2}}{\left|\alpha^{\prime}(t)\right|^{4}}$ fonksiyonu $\mathcal{M} I D_{1}$-invaryanttır.

5. Regüler Parametrik Eğrilerin (Yolların) Tam İnvaryantlar Sistemi ve Regüler Parametrik Eğrilerin (Yolların) Teklik Teoremleri

$G=\mathcal{M} I D_{1}^{+}$olsun. Bu bölümde iki parametrik eğrinin denklik koşullarını parametrik eğrilerin $d$-regüler olması durumunda ifade eden aşağıdaki teoremi ispatlayarak başlayacağız.

Teorem 5.1. $\alpha(t)$ ve $\beta(t) \quad \mathbb{R}^{2}$ 'de $d$-regüler $T$-yollar olsun. $\alpha(t) \stackrel{G}{\sim} \beta(t)$ dir. $\Leftrightarrow \quad \forall t \in T$ için

$\left|F \alpha^{\prime}(t)\right|=a x^{\prime}(t)$ ve 


$$
\begin{aligned}
\frac{\left|\alpha^{\prime \prime}(t)\right|}{\left|\alpha^{\prime}(t)\right|} & =\frac{\left|\beta^{\prime \prime}(t)\right|}{\left|\beta^{\prime}(t)\right|} \\
\frac{\left[\alpha^{\prime}(t) \alpha^{\prime \prime}(t)\right]}{\left|\alpha^{\prime}(t)\right|^{2}} & =\frac{\left[\beta^{\prime}(t) \beta^{\prime \prime}(t)\right]}{\left|\beta^{\prime}(t)\right|^{2}}
\end{aligned}
$$

dir. Ayrıca; $U \in I D_{1}^{+}, C \in \mathbb{R}^{2}$ olmak üzere; bir tek $F \in \quad \mathcal{M} I D_{1}^{+}$vardır öyle ki $\beta(t)=F \alpha(t)=U \alpha(t)+C$ dir. Ve burada

$$
U=\left(\begin{array}{cc}
\frac{\left|\beta^{\prime}(t)\right|}{\left|\alpha^{\prime}(t)\right|} & 0 \\
\frac{\left[\alpha^{\prime}(t) \beta^{\prime}(t)\right]}{\left|\alpha^{\prime}(t)\right|^{2}} & \frac{\left|\beta^{\prime}(t)\right|}{\left|\alpha^{\prime}(t)\right|}
\end{array}\right)
$$

ve $C=\beta(t)-U \alpha(t)$ dir. Burada $U$ ve $C$ sabittir.

İspat: $(\Rightarrow) \alpha(t) \stackrel{G}{\sim} \beta(t)$ kabul edelim.

Teorem 3.1. 'den $\forall t \in T$ ve $F \in \mathcal{M} I D_{1}^{+}$, $H \in D_{1}$ için $\beta(t)=H \alpha(t)+C$ dir. Buradan $\beta^{\prime}(t)=H \alpha^{\prime}(t)$ olur. $\alpha(t)$ ve $\beta(t) d$-regüler $T$-yollar olduğundan $\left|\alpha^{\prime}(t)\right| \neq 0$ ve $\left|\beta^{\prime}(t)\right| \neq 0$ dir. O halde; $\forall t \in T$ için Önerme 2.3. ten $\left(\alpha^{\prime}(t)\right)^{-1}$ ve $\left(\beta^{\prime}(t)\right)^{-1}$ vardır. Önerme 3.2. de $A=\beta^{\prime}(t)$ ve $B=\beta^{\prime \prime}(t)$ alalım. O halde;

$$
\frac{\beta^{\prime \prime}(t)}{\beta^{\prime}(t)}=\frac{\left|\beta^{\prime \prime}(t)\right|}{\left|\beta^{\prime}(t)\right|}+\varepsilon \frac{\left[\beta^{\prime}(t) \beta^{\prime \prime}(t)\right]}{\left|\beta^{\prime}(t)\right|^{2}}
$$

elde edilir. Benzer şekilde;

$$
\frac{\alpha^{\prime \prime}(t)}{\alpha^{\prime}(t)}=\frac{\left|\alpha^{\prime \prime}(t)\right|}{\left|\alpha^{\prime}(t)\right|}+\varepsilon \frac{\left[\alpha^{\prime}(t) \alpha^{\prime \prime}(t)\right]}{\left|\alpha^{\prime}(t)\right|^{2}}
$$

elde edilir. $\beta^{\prime}(t)=H \alpha^{\prime}(t)$ ve $\beta^{\prime \prime}(t)=H \alpha^{\prime \prime}(t)$ den $\forall t \in T$ için $\frac{\beta^{\prime \prime}(t)}{\beta^{\prime}(t)}=\frac{\alpha^{\prime \prime}(t)}{\alpha^{\prime}(t)}$ sağlanır. $\mathrm{Bu}$ eşitlik, (*) ve (**)' dan $\frac{\left|\alpha^{\prime \prime}(t)\right|}{\left|\alpha^{\prime}(t)\right|}=\frac{\left|\beta^{\prime \prime}(t)\right|}{\left|\beta^{\prime}(t)\right|}$, $\frac{\left[\alpha^{\prime}(t) \alpha^{\prime \prime}(t)\right]}{\left|\alpha^{\prime}(t)\right|^{2}}=\frac{\left[\beta^{\prime}(t) \beta^{\prime \prime}(t)\right]}{\left|\beta^{\prime}(t)\right|^{2}}$ elde edilir.

$(\Leftarrow)$ Kabul edelim ki; (1) sağlansın.

$$
\begin{aligned}
& \frac{\left|\alpha^{\prime \prime}(t)\right|}{\left|\alpha^{\prime}(t)\right|}=\frac{\left|\beta^{\prime \prime}(t)\right|}{\left|\beta^{\prime}(t)\right|}, \frac{\left[\alpha^{\prime}(t) \alpha^{\prime \prime}(t)\right]}{\left|\alpha^{\prime}(t)\right|^{2}}=\frac{\left[\beta^{\prime}(t) \beta^{\prime \prime}(t)\right]}{\left|\beta^{\prime}(t)\right|^{2}}, \\
& (*) \text { ve } \quad\left(^{* *}\right) \text { 'dan } \frac{\beta^{\prime \prime}(t)}{\beta^{\prime}(t)}=\frac{\alpha^{\prime \prime}(t)}{\alpha^{\prime}(t)} \text { sağlanır. }
\end{aligned}
$$

Buradan $\beta^{\prime \prime}(t)\left(\beta^{\prime}(t)\right)^{-1}-\alpha^{\prime \prime}(t)\left(\alpha^{\prime}(t)\right)^{-1}=0$.

Bu eşitlik kullanılarak;

$\frac{d\left(\beta^{\prime}(t)\left(\alpha^{\prime}(t)\right)^{-1}\right)}{d t}=$

$=\beta^{\prime \prime}(t)\left(\alpha^{\prime}(t)\right)^{-1}-\beta^{\prime}(t) \alpha^{\prime \prime}(t)\left(\alpha^{\prime}(t)\right)^{-2}$

$=\left(\beta^{\prime \prime}(t)-\beta^{\prime}(t) \alpha^{\prime \prime}(t)\left(\alpha^{\prime}(t)\right)^{-1}\right)\left(\alpha^{\prime}(t)\right)^{-1}$

$=\beta^{\prime}(t)\left(\beta^{\prime \prime}(t)\left(\beta^{\prime}(t)\right)^{-1}-\alpha^{\prime \prime}(t)\left(\alpha^{\prime}(t)\right)^{-1}\right)\left(\alpha^{\prime}(t)\right)^{-1}$

$=0$

elde edilir. Buradan; $\beta^{\prime}(t)\left(\alpha^{\prime}(t)\right)^{-1}$ sabittir. $K=\beta^{\prime}(t)\left(\alpha^{\prime}(t)\right)^{-1}$ olarak alalım. $K$ sabit olduğundan $|K|$ de sabit olur. $\forall t \in T$ için $\left|\alpha^{\prime}(t)\right| \neq 0,\left|\beta^{\prime}(t)\right| \neq 0$ kullanılırsa

Önerme 2.3. ten $|K|=\left|\beta^{\prime}(t)\left(\alpha^{\prime}(t)\right)^{-1}\right|$ $=\left|\beta^{\prime}(t)\right|\left|\left(\alpha^{\prime}(t)\right)^{-1}\right|=\frac{\left|\beta^{\prime}(t)\right|}{\left|\alpha^{\prime}(t)\right|} \neq 0$ ‘dır. Böylece; $|K|$ hem sabittir hem de $|K| \neq 0$ 'dır. Dolayısıyla $K \in D_{1}$ dir.

$\beta^{\prime}(t)=\beta^{\prime}(t)\left(\alpha^{\prime}(t)\right)^{-1} \alpha^{\prime}(t)=\left(\beta^{\prime}(t)\left(\alpha^{\prime}(t)\right)^{-1}\right) \alpha^{\prime}(t)$ $\beta^{\prime}(t)=K \alpha^{\prime}(t)$ elde edilir. Teorem 4.1. (i) kullanılarak

$$
K=\beta^{\prime}(t)\left(\alpha^{\prime}(t)\right)^{-1}=\frac{\left|\beta^{\prime}(t)\right|}{\left|\alpha^{\prime}(t)\right|}+\varepsilon \frac{\left[\alpha^{\prime}(t) \beta^{\prime}(t)\right]}{\left|\alpha^{\prime}(t)\right|^{2}}
$$

elde edilir. $K=U$ olduğu görülür. $K$ sabit olduğundan $U$ sabittir. Buradan $\beta^{\prime}(t)=U \alpha^{\prime}(t)=K \alpha^{\prime}(t)$ yazilır. Buradan da integral alarak $\beta(t)=U \alpha(t)+C$ elde edilir. Böylece; $\alpha(t)$ ile $\beta(t)$ G-denktir.

$F \in \mathcal{M} I D_{1}^{+}$' nın tekliğini gösterelim. Bunun için $U \in I D_{1}^{+}$ve $C \in \mathbb{R}^{2}$ 'nin tekliğini göstermemiz gerekir. $\mathrm{O}$ halde; $F \in \mathcal{M} I D_{1}^{+}$ olduğundan $\quad \beta(t)=F \alpha(t)=U \alpha(t)+C \quad$, $U \in I D_{1}^{+}, \quad C \in \mathbb{R}^{2} \quad$ sağlanır. Diyelim ki; 
$E \in \mathbb{R}^{2}$ olmak üzere; $V \in I D_{1}^{+}$var ve $\beta(t)=V \alpha(t)+E$ olsun. $\quad \beta^{\prime}(t)=V \alpha^{\prime}(t)$ sağlanır. $\quad\left|\alpha^{\prime}(t)\right| \neq 0 \quad$ ve $\quad \beta^{\prime}(t)=V \alpha^{\prime}(t)$ kullanılarak $V=\beta^{\prime}(t)\left(\alpha^{\prime}(t)\right)^{-1}=K$. Böylece; $V=K=U$. Dolayısiyla; U tektir. $C=\beta(t)-U \alpha(t) \quad$ olarak alalım. $U$ 'nun tekliğinden $C=\beta(t)-U \alpha(t)=E$. Böylece; $U$ ve $C$ 'nin de tekliğinden $F \in \mathcal{M} I D_{1}^{+}$, nin tekliği de elde edilir.

Tanım 5.1. $\mathbb{R}^{2}$ 'de $\alpha(t)$ bir $T$-yol olmak üzere; $\forall t \in T$ için $\left[\alpha^{\prime}(t) \alpha^{\prime \prime}(t)\right]=0$ ise $\alpha(t)$ $T$-yoluna tam dejenere denir.

$\alpha(t)$ ve $\beta(t) \mathbb{R}^{2}$ 'de $T$-yollar olsun.

$\alpha(t)$ tam dejenere ve $G=\mathcal{M} I D_{1}^{+}$olmak üzere;

$\alpha(t) \stackrel{G}{\sim} \beta(t)$ ise $\beta(t)$ de tam dejeneredir.

Teorem 5.2. $\alpha(t)$ ve $\beta(t) \quad \mathbb{R}^{2}$ 'de tam dejenere $d$-regüler T-yollar olsun.

$G=\mathcal{M} I D_{1}$ olmak üzere; $\alpha(t) \stackrel{G}{\sim} \beta(t)$ dir. $\Leftrightarrow$ $\forall t \in T$ için

$$
\frac{\left|\alpha^{\prime \prime}(t)\right|}{\left|\alpha^{\prime}(t)\right|}=\frac{\left|\beta^{\prime \prime}(t)\right|}{\left|\beta^{\prime}(t)\right|}
$$

dir. Ayrica; $U_{1}, U_{2} \in \mathcal{M} I D_{1}^{+}, \quad C_{1}, C_{2} \in \mathbb{R}^{2}$ olmak üzere sadece iki tane $F=F_{1}, F_{2} \in \mathcal{M}$ $I D_{1}$ vardır öyle ki $\beta(t)=F_{1} \alpha(t)=U_{1} \alpha(t)+C_{1}$ ve $\beta(t)=F_{2} \alpha(t)=\left(U_{2} W\right) \alpha(t)+C_{2}$. Burada

$$
\begin{gathered}
U_{1}=\left(\begin{array}{cc}
\frac{\left|\beta^{\prime}(t)\right|}{\left|\alpha^{\prime}(t)\right|} & 0 \\
\frac{\left[\alpha^{\prime}(t) \beta^{\prime}(t)\right]}{\left|\alpha^{\prime}(t)\right|^{2}} & \frac{\left|\beta^{\prime}(t)\right|}{\left|\alpha^{\prime}(t)\right|}
\end{array}\right) \text { ve } \\
U_{2}=\left(\begin{array}{cc}
\frac{\left|\beta^{\prime}(t)\right|}{\left|W \alpha^{\prime}(t)\right|} & 0 \\
\frac{\left[W \alpha^{\prime}(t) \beta^{\prime}(t)\right]}{\left|W \alpha^{\prime}(t)\right|^{2}} & \frac{\left|\beta^{\prime}(t)\right|}{\left|W \alpha^{\prime}(t)\right|}
\end{array}\right)
\end{gathered}
$$

dir. $C_{1}=\beta(t)-U_{1} \alpha(t), \quad C_{2}=\beta(t)-U_{2} \alpha(t)$ $\operatorname{dir} . U_{1}, U_{2}, C_{1}, C_{2}$ sabittir.
İspat: $\quad(\Rightarrow) \quad G=\mathcal{M} I D_{1}$ olmak üzere; $\alpha(t) \stackrel{G}{\sim} \beta(t)$ kabul edelim. $\alpha(t)$ ve $\beta(t) \quad \mathbb{R}^{2}$ 'de tam dejenere $d$-regüler T-yollar olduğundan $\forall t \in T \quad$ için $\quad\left|\alpha^{\prime}(t)\right| \neq 0 \quad$ ve $\left|\beta^{\prime}(t)\right| \neq 0$ 'dır. Böylece; $\forall t \in T$ için $\frac{\left|\alpha^{\prime \prime}(t)\right|}{\left|\alpha^{\prime}(t)\right|}$ ve $\frac{\left|\beta^{\prime \prime}(t)\right|}{\left|\beta^{\prime}(t)\right|}$ vardır. Teorem 4.2. (i) 'den $\frac{\left|\alpha^{\prime \prime}(t)\right|}{\left|\alpha^{\prime}(t)\right|}$ $\mathcal{M} I D_{1}$-invaryanttır. Teorem 5.1. 'den (3) elde edilir.

$(\Leftarrow)$ Kabul edelim ki; (3) sağlansın. $\alpha(t)$ ve $\beta(t) \quad \mathbb{R}^{2}$ 'de $d$-regüler $T$-yollar olduğundan $\forall t \in T$ için $\left|\alpha^{\prime}(t)\right| \neq 0$ ve $\left|\beta^{\prime}(t)\right| \neq 0 \quad$ 'dır.

Böylece; $\forall t \in T \quad$ için $\frac{\left[\alpha^{\prime}(t) \alpha^{\prime \prime}(t)\right]}{\left|\alpha^{\prime}(t)\right|^{2}} \quad$ ve $\frac{\left[\beta^{\prime}(t) \beta^{\prime \prime}(t)\right]}{\left|\beta^{\prime}(t)\right|^{2}}$ vardır. $\alpha(t)$ ve $\beta(t) \mathbb{R}^{2}$ 'de tam dejenere $T$-yollar olduğundan $\forall t \in T$ için $\left[\alpha^{\prime}(t) \alpha^{\prime \prime}(t)\right]=\left[\beta^{\prime}(t) \beta^{\prime \prime}(t)\right]=0 \quad$ elde edilir. Buradan

$$
\frac{\left[\alpha^{\prime}(t) \alpha^{\prime \prime}(t)\right]}{\left|\alpha^{\prime}(t)\right|^{2}}=\frac{\left[\beta^{\prime}(t) \beta^{\prime \prime}(t)\right]}{\left|\beta^{\prime}(t)\right|^{2}}=0
$$

elde edilir.

(5) ve (3) denklemleri kullanılarak

Teorem 5.1.' de yer alan denklemler elde edilir. Teorem 5.1. kullanılarak bir tek

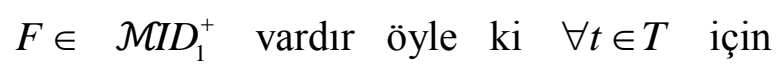
$\beta(t)=F \alpha(t)$ 'dir. Buradan görülür ki; bir tek $U_{1} \in \mathcal{M} I D_{1}^{+}$ve bir tek $C_{1} \in \mathbb{R}^{2}$ vardir ve $U_{1}=U \in \quad \mathcal{M I D}_{1}^{+} \quad$ 'dır. Öyle ki; $\beta(t)=F \alpha(t)=U_{1} \alpha(t)+C_{1} \quad$ ve $C_{1}=\beta(t)-U_{1} \alpha(t)$ 'dir. Teorem 5.1.' den $U_{1}$ ve $C_{1}$ sabittir.

$W \alpha(t) T$-yol olsun. Önerme 3.4. (i) 'den $\frac{\left|\alpha^{\prime \prime}(t)\right|}{\left|\alpha^{\prime}(t)\right|} W$-invaryanttır. Böylece; $\forall t \in T$ için 


$$
\frac{\left|W \alpha^{\prime \prime}(t)\right|}{\left|W \alpha^{\prime}(t)\right|}=\frac{\left|\alpha^{\prime \prime}(t)\right|}{\left|\alpha^{\prime}(t)\right|} .
$$

'dir. (6) ve (3) kullanılarak,

$$
\frac{\left|W \alpha^{\prime \prime}(t)\right|}{\left|W \alpha^{\prime}(t)\right|}=\frac{\left|\beta^{\prime \prime}(t)\right|}{\left|\beta^{\prime}(t)\right|} \ldots
$$

elde edilir. Önerme 3.4. (i)' de $\forall t \in T$ için $\left|W \alpha^{\prime}(t)\right|^{2}=\left|\alpha^{\prime}(t)\right|^{2}, \quad \operatorname{det}(W)=-1 \quad$ ve

kullanılarak;

$$
\begin{aligned}
& \frac{\left[W \alpha^{\prime}(t) W \alpha^{\prime \prime}(t)\right]}{\left|W \alpha^{\prime}(t)\right|^{2}}=\frac{\operatorname{det}(W)\left[\alpha^{\prime}(t) \alpha^{\prime \prime}(t)\right]}{\left|\alpha^{\prime}(t)\right|^{2}} \\
& =-\frac{\left[\alpha^{\prime}(t) \alpha^{\prime \prime}(t)\right]}{\left|\alpha^{\prime}(t)\right|^{2}}=-\frac{\left[\beta^{\prime}(t) \beta^{\prime \prime}(t)\right]}{\left|\beta^{\prime}(t)\right|^{2}}=0 .
\end{aligned}
$$

Böylece;

$$
\frac{\left[W \alpha^{\prime}(t) W \alpha^{\prime \prime}(t)\right]}{\left|W \alpha^{\prime}(t)\right|^{2}}=\frac{\left[\beta^{\prime}(t) \beta^{\prime \prime}(t)\right]}{\left|\beta^{\prime}(t)\right|^{2}}
$$

elde edilir. $\forall t \in T$ için (7) ve (8) sistemi elde edilir. Teorem 5.1. kullanılarak bir tek

$F \in \mathcal{M} I D_{1}^{+}$vardır öyle ki $\forall t \in T$ için $\beta(t)=F(W \alpha(t))$ 'dir. $F \in \mathcal{M} I D_{1}^{+}{ }^{\prime}$ nin

tekliğinden

$\forall t \in T$ için $\beta(t)=F \alpha(t)=\left(U_{2} W\right) \alpha(t)+C_{2}$ 'yi sağlayan bir tek $U_{2} \in \mathcal{M} I D_{1}^{+}$ve bir tek $C_{2} \in \mathbb{R}^{2}$ vardir. Burada $U_{2} \quad$ (4) şeklindedir ve $C_{2}=\beta(t)-\left(U_{2} W\right) \alpha(t)$ 'dir. Teorem 5.1.'den $U_{2} \in \mathcal{M} I D_{1}^{+}$ve $C_{2} \in \mathbb{R}^{2}$ sabittir.

$F \in \mathcal{M} I D_{1}$ alalım. Öyle ki $\beta(t)=F \alpha(t)$ 'dir. $U_{1}, U_{2} \in I D_{1}^{+}$ve $C_{1}, C_{2} \in \mathbb{R}^{2}$ olmak üzere; $F \alpha(t)=U_{1} \alpha(t)+C_{1}$ veya

$$
F \alpha(t)=\left(U_{2} W\right) \alpha(t)+C_{2}
$$

olduğunu

gösterelim. $L \in I D_{1}, C_{3} \in \mathbb{R}^{2}$ olmak üzere; $\beta(t)=F \alpha(t)=L \alpha(t)+C_{3}$ alalım. $L \in I D_{1}$ olduğundan $L \in I D_{1}^{+}$veya $L \in I D_{1}^{-}$olur. Kabul edelim ki $L \in I D_{1}^{+}$olsun. Teorem 5.1.' deki teklik kullanılarak, $L=U_{1} \quad$ ve $C_{1}=C_{3}=\beta(t)-U_{1} \alpha(t)$ elde edilir.
Kabul edelim ki; $L \in I D_{1}^{-}$olsun. $P \in I D_{1}^{+}$ olmak üzere; $L=P W \quad$ ve $C_{3}=\beta(t)-P W \alpha(t)$ şeklinde olur. Buradan $\beta(t)=(P W) \alpha(t)+C_{3}=P(W \alpha(t))+C_{3} \quad$ elde edilir. Böylece; $\beta(t)$ ve $W \alpha(t)$

T-yolları $\mathcal{M} I D_{1}^{+}$-denktirler. Teorem 4.1.' deki teklik kullanılarak $\forall t \in T$ için $P=U_{2}$ ve $C_{2}=C_{3}=\beta(t)-\left(U_{2} W\right) \alpha(t)$ elde edilir.

(a) ve (d) durumunda, $U_{1} \in \mathcal{M} I D_{1}^{+}$, $C_{1}=\beta(t)-U_{1} \alpha(t) \quad$ olmak üzere; $F \alpha(t)=U_{1} \alpha(t)+C_{1}$ sağlanır. Burada $U_{1}$ (2) şeklindedir. (b) ve (c) durumunda, $U_{2} \in \mathcal{M}$ $I D_{1}^{+}, \quad C_{2}=\beta(t)-U_{2} W \alpha(t) \quad$ olmak üzere; $F \alpha(t)=U_{2} W \alpha(t)+C_{2}$ sağlanır. Burada $U_{2}$ (4) şeklindedir. $U_{1}, U_{2}, C_{1}, C_{2} t \in T$ sabittir.

\section{Sonuç}

$\mathrm{Bu}$ çalışmada, kullanılan yöntem ile parametrik eğrilerin G-denklik problemi ile ilgili sonuçlar elde edilmiştir. Kullanılan yöntem ve sonuçlar dual parametrik eğrilerin (yolların) ve dual eğrilerin invaryant teorideki uygulamalarında yararlı olacaktır.

\section{Kaynaklar}

Khadijev D., Oren I., Peksen O. 2018. 'Global invariants of paths and curves for the group of all linear similarities in the two-dimensional Euclidean space', International Journal of Geometric Methods in Modern Physics Vol. 15.

Khadjiev D.,1988, "Application of the Invariant Theory to the Differential Geometry of Curves"' Fan Publisher, Tashkent.

Klein, F. 1872. "Vergleichende Betrachtungen Über Neuere Geometrische Forschungen",Erlangen: Verlag.

TOMAR, M. 2012. 'Applications of dual numbers and dual numbers to two- 
dimensional dual geometry', Master's Thesis, Science Institute, Trabzon. 